\title{
Theoretical Study of the Influence of the Morphology in Polymer-Based Devices Functioning
}

\author{
Hélder M. C. Barbosa ${ }^{1, a, *}$, Marta M. D. Ramos ${ }^{1, b}$ \\ ${ }^{1}$ Departamento de Física, Universidade do Minho, \\ Campus de Gualtar, 4710-057 Braga, Portugal \\ Tel.: +351253604 330; Fax: +351253678981 \\ ${ }^{a}$ helder@fisica.uminho.pt (H. M. C. Barbosa) \\ ${ }^{\mathrm{b}}$ marta@fisica.uminho.pt (M. M. D. Ramos)
}

*Corresponding author

\begin{abstract}
It is well known that the morphology of polymer-based optoelectronic devices can influence their efficiency, since the ways that polymer chains pack inside the active layer can influence not only the charge transport but also the optic properties of the device. By using a mesoscopic model we carried out computer experiments to study the influence of the polymer morphology on the processes of charge injection, transport, recombination and collection by the electrodes opposite to those where the injection of bipolar charge carriers take place. Our results show that for polymer layers where the conjugated segments have perpendicular and random orientation relative to the electrodes surface, the competition between charge collection and charge recombination is affected when the average conjugation length of the polymer strands increase. This effect is more pronounced with the increase of the potential barrier at polymer/electrode interfaces that limit charge injection and increase charge collection. For these molecular arrangements the intra-molecular charge transport plays a major role in device performance, being this effect negligible when the polymer molecules have their axis parallel to the electrodes. Although the polymer morphology modelled in this work is far from real, we believe that our model can give some insights on the role of the microstructure on the functioning of polymer-based devices.
\end{abstract}


Keywords: Mesoscopic modelling, PPV, conjugation length, chain orientation, polymer morphology

\section{Introduction}

Conjugated polymers are unique materials that exhibit the optoelectronic properties of inorganic semiconductors as well as the mechanical properties and processing advantages of plastics. Nevertheless, the possibility of using this kind of materials in light emitting diodes (LEDs), solar cells or transistors is limited because the efficiency of these devices depends strongly on the polymer film morphology and the type of electrodes used.

After deposition, the polymer layer exhibits a unique morphology where the conjugated chains tend to aggregate. The torsion of the chain backbone tend to disrupt the conjugation, leading to the creation of conjugated segments with varied lengths, that behave like independent molecules [1]. This chain-packing form a nanodomain where the conjugated segments are well oriented to each other. The orientation of these nanodomains relative to the electrodes surface depends on the deposition conditions used. In a spin-coated layer the conjugated segments are mainly oriented parallel to the electrodes surface [2] while in an ink-jet layer the conjugated segments have perpendicular orientation [1]. After deposition, the annealing process allows a reorganization of the polymer chains in which the segments acquire a random orientation [3]. Experimental results show that the orientation of the aggregates can affect not only charge mobility [4] but also their optical properties [5], whereas theoretical results show that the molecular properties of each segment depend on their length [6].

For the case of polymer LED devices a balanced charge injection is required to achieve a good performance [7]. In this way it is necessary to reduce the potential barrier height that exists between the electrodes and the polymer and to improve charge transport towards the bulk to reduce space charge effects for the process of charge injection.

The purpose of this work is to get some insight on the influence of changing the average conjugation length of the polymer layer as well as the orientation of the conjugated segments relative to the electrodes surface on the functioning of a polymer 
light emitting diode (PLED), with a single p-(polyphenylene vinylene) (PPV) layer as the active component, and several potential barrier heights for charge injection.

\section{Model and Simulation Details}

Polymer layers present a microstructure where the polymer chains can be seen as a connection of stiff-chain segments with different lengths and orientations, creating an anisotropic system. Each segment works as a localized site where charge injection and transport between segments is temperature activated, in a process known as hopping.

Quantum mechanical calculations show that when a charge is injected into a polymer strand it will stay in the middle of it because this is the position energetic more favourable. Nevertheless, if the electric field along the strand axis is above a certain threshold, which depends on charge sign, the charge can move to one of the strand ends [8]. This intra-molecular charge mobility is faster than the mobility of the charge between neighbouring strands (inter-molecular mobility) and can strongly influence charge percolation through the polymer network [9]. Depending on the orientation of the polymer strands or their length, and thus on the microstructure of the polymer layer, the influence of both intra-molecular or inter-molecular charge transport on polymer LED performance can be quite different.

By adopting a mesoscopic approach we construct a model that considers both inter-molecular and intra-molecular charge transport to study the influence of the polymer morphology, the molecular properties and the electrode work function in charge injection, transport and recombination.

By using the same strategy as reported before [10] the polymer networks where built between two planar electrodes by placing stiff-chain segments with lengths taken from a Gaussian distribution with a mean values that vary from 5 to 7 monomers. To take into account the different orientations of the polymer segment axis relative to the electrodes surface mentioned above, we construct PPV films where the straight rigid segments are placed parallel, perpendicular and randomly oriented relative to the electrodes surface and with a minimum inter-molecular distance of $0.650 \mathrm{~nm}$ based on self-consistent quantum molecular dynamics calculations [11]. 
Our model takes into account the competition between the processes of charge injection, transport, trapping, recombination and collection by the electrodes, using a generalized Monte Carlo method [12].

The processes of charge (hole or electron) injection into polymer strands and inter-molecular charge transport are achieved by considering the hopping probability between the electrodes and the polymer strands, and between neighbouring strands. The hopping probability considers the distance between hopping sites, as well as the potential barrier between them. In the case of charge injection and in the absence of an applied electric field, the potential barrier height (zero-field barrier height) is equal to the difference between cathode/anode work function and the electron affinity (EA)/ionization potential (IP) (i.e. the molecular properties) of the polymer strand. By changing the electrode work function it is possible to control the zero-field barrier height and thus charge injection. When an electric field is applied, the potential barrier height for charge injection is changed due to the local electric field (i.e the sum of the applied electric field, the field due to the spatial distribution of electrons and holes in transit or trapped within polymer network and the field that results from the electrodes polarization). For intermolecular charge transport, the zero-field potential barrier is equal to the difference of the electron affinity/ionization potential between the strands involved in the hopping of an electron/hole and this barrier height is also affect by the local electric field.

After injection, charges can percolate through the polymer network. During this process a charge can be stored or trapped in a strand until the local electric field allows it to hop to a neighbour strand, it can meet a charge of opposite sign in the same strand, or it can be collect by the electrode opposite to the injection electrode. When two charges of opposite sign meet in the same polymer strand a recombination event can occur if the local electric field along the conjugated segment axis is not strong enough to avoid the movement of both charges towards each other.

\section{Results and Discussion}

To get some insight on the relationship between electrode work function and the molecular arrangements of the polymer layer in the LED performance, we simulate bipolar charge injection and transport in PPV layers, where the polymer strands have the 
three different orientations described previously. To get a balance in the number of electrons and holes injected, we have changed the electrodes work function so the average zero-field barrier height at both polymer/electrode interfaces (i.e. the difference between the average ionization potential/electron affinity, considering the distribution of chain lengths near the electrodes, and the anode/cathode work function) remain the same. All simulations were performed for an external electric field of $3 \mathrm{MV} / \mathrm{cm}$, above the threshold for intra-molecular mobility of electrons and holes [8], and we have changed the electrodes work function so the average zero-field barrier height varies from $0.2 \mathrm{eV}$ to $0.8 \mathrm{eV}$.

[Insert Figure 1] Fig. 1 shows the total amount of injected charge in layers with different polymer morphology as a function of the average conjugation length and for different zero-field barrier heights at both polymer/electrode interfaces. These results where obtained at the end of the simulations when the steady state is reached. As we can see, the amount of charges injected in the parallel morphology is smaller than for random and perpendicular morphologies. This is due to the fact that for the parallel morphology the number of conjugated segments near the electrodes surface, and thus the number of injection sites, is smaller than for the other two morphologies, which limits the amount of charge injected. On the other hand, the contribution of intra-molecular charge mobility to charge transport towards the electrode opposite to the injection electrode is insignificant and so charge transport for this morphology is mainly due to charge hopping between strands. Since this is the slowest step in charge transport, charges will stay longer near the electrode/polymer interfaces and therefore the effect of spatial distribution of the injected charges is more pronounced for the parallel morphology than for the other two morphologies. It is interesting to observe that the increase of the electrode work function, and thus the zero-field barrier height, reduces the amount of charge injected being this effect more pronounced for the perpendicular morphology. With the increase of the zerofield barrier height the number of polymer strands available for charge injection is reduced since not all the conjugated segments exhibit the same molecular properties, which is valid for all morphologies. Due to their orientation relative to the electrodes surface, all the monomers of a segment are suitable for charge injection in the parallel and random morphologies but the same do not happen for the perpendicular morphology 
where just the monomers near the electrode surface can work like an injection site. The fact that there is more charge injected in layers with random morphology than in layers with parallel morphology is also due to space charge effects that are more pronounced for the parallel morphology than for the random morphology, which limits the amount of charge injected.

The increase of the average conjugation length seems to increase the amount of charge injected for random and perpendicular morphologies, and leads to a slight decrease of injection for the parallel morphology. The increase of the average conjugation length reduces the number of chains near the electrodes surface and so the number of injection sites in layers with parallel morphology, whereas increases intramolecular charge mobility in layers with random and perpendicular morphologies and, consequently, the charge transport through the polymer layer with such morphologies.

[Insert Figure 2] The role of the intra-molecular charge mobility in polymer LEDs performance becomes evident when we analyse charge collection by the electrodes. Fig. 2 shows the fraction of the injected charges that are collected by the electrodes opposite to those where they were injected for all the morphologies considered in this work. The amount of charges collected by both electrodes is negligible for the layers with parallel morphology, being higher for layers with perpendicular morphology.

For the parallel morphology, the charge transport is mainly due to hopping between strands and since this is the slowest step in charge transport, it will lead to an increase of charge transit time inside the polymer network. For the random and perpendicular morphologies the possibility of intra-molecular charge mobility reduces the charge transit time inside the polymer network, being this effect more pronounced as the average conjugation length of the polymer strands increase. With the increase of the zerofield barrier height at polymer/electrode interfaces there is a decrease on the number of charges in transit inside the polymer network.

[Insert Figure 3] Fig. 3 shows the fraction of injected charges that undergoes recombination for all the morphologies considered. Comparing Fig. 2 and Fig. 3, we see that charge recombination and charge collection by the electrodes show an opposite behaviour because the amount of charge stored within the polymer layer does not change significantly as the average conjugation length increases and decreases with the increase 
of the zero-field barrier height at both polymer/electrode interfaces. The increase of both the zero-field barrier height and the average conjugation length of the polymer chains reduce the fraction of the injected charges that undergo recombination in the random and perpendicular morphologies, but remains roughly constant for the parallel morphology.

[Insert Figure 4] Although the amount of charges stored in the polymer layers depend on their morphology, it seems that the probability of two charges meet at the same strand and recombine depends more on their transit time inside the polymer network than on the concentration of charge carriers. Comparing Fig. 3 and Fig. 4, we can see that the layers with parallel morphology have the smallest carrier concentration but the higher fraction of charges that undergo recombination. The opposite behaviour occurs for the layers with perpendicular morphology.

\section{Conclusions}

Although most has been learned about the influence of the deposition parameters in polymer layer morphology there is not a clear picture for the correlation between the morphology of the polymer layer and the LED efficiency. By using a mesoscopic model we try to shed some light on the influence of the molecular arrangements in the processes involved in polymer LED functioning. Our results show that the intra-molecular mobility plays a major role in device functioning with perpendicular and random molecular arrangements. When the polymer/electrode contacts are ohmic (small zero-field barrier heights) the polymer layer with perpendicular morphology seems to be the most efficient for charge injection whereas the random morphology is more efficient for charge injection when the contacts are non-ohmic (height zero field barrier heights). In both cases charge injection and the collected charge increases with the increase of the average conjugation length. Despite the fact the polymer layer with parallel morphology is more inefficient for charge injection as compared with perpendicular and random morphologies, our results show this is quite efficient for charge recombination independent of the average conjugation length of the polymer strands and height of the zero-field barrier at polymer electrode interfaces. 


\section{Acknowledgement}

This work is part of the research projects POCTI/CTM/41574/2001 and CONC-

REEQ/443/EEI/2005, approved by the Portuguese Foundation for Science and Technology (FCT) and support by the European Community Fund FEDER. One of us (H.M.C.B.) is also indebted to FCT for financial support under $\mathrm{PhD}$ grant $\mathrm{N}^{\circ}$ SFRH/BD/22143/2005.

\section{References}

[1] B. G. SUMPTER, P. KUMAR, A. MEHTA, M. D. BARNES, W. A. SHELTON and R. J. HARRISON, J. Phys. Chem. B 109 (2005) 7671.

[2] C. Y. YANG, F. HIDE, M. A. DIAZ-GARCIA, A. J. HEEGER and Y. CAO, Polymer 39 (1998) 2299.

[3] J. KIM, J. LEE, C. W. HAN, N. Y. LEE and I. J. CHUNG, Appl. Phys. Lett. 82 (2003) 4238.

[4] H. F. MENG, B. L. CHEN, K. C. TZENG and S. F. HORNG, Appl. Phys. Lett. 88 (2006) 3.

[5] K. KOYNOV, A. BAHTIAR, T. AHN, R. M. CORDEIRO, H. H. HORHOLD and C. BUBECK, Macromolecules 39 (2006) 8692.

[6] A. M. STONEHAM, M. M. D. RAMOS, A. M. ALMEIDA, H. M. G. CORREIA, R. M. RIBEIRO, H. NESS and A. J. FISHER, J. Phys.-Condes. Matter 14 (2002) 9877.

[7] P. W. M. BLOM and M. J. M. DE JONG, Philips J. Res. 51 (1998) 479.

[8] A. M. ALMEIDA, M. M. D. RAMOS and H. G. CORREIA, Comput. Mater. Sci. 27 (2003) 128.

[9] A. M. STONEHAM and M. M. D. RAMOS, J. Phys.-Condes. Matter 13 (2001) 2411.

[10] M. M. D. RAMOS, H. M. G. CORREIA, R. M. RIBEIRO and A. M. STONEHAM, Synth. Met. 147 (2004) 281.

[11] M. M. D. RAMOS, A. M. ALMEIDA, H. M. G. CORREIA, R. M. RIBEIRO and A. M. STONEHAM, Appl. Surf. Sci. 238 (2004) 438.

[12] M. M. D RAMOS and H. M. G. CORREIA, J. Phys.-Condes. Matter 18 (2006) S429. 


\section{Figure captions:}

Fig. 1 - Total number of charges (electrons and holes) injected into the PPV layers with random, parallel and perpendicular morphologies, as a function of the average conjugated segment length, when the zero-field barrier height at both polymer/electrode interfaces is $0.2 \mathrm{eV}$ (squares), $0.4 \mathrm{eV}$ (circles), $0.6 \mathrm{eV}$ (triangles) and $0.8 \mathrm{eV}$ (stars). The lines are just a guide to the eyes.

Fig. 2 - Proportion of the injected charges in the PPV layers, with random, parallel and perpendicular morphologies, that are collect by the electrodes opposite to the injection electrode as a function of the average conjugated segment length, when the zero-field barrier height at both polymer/electrode interfaces is $0.2 \mathrm{eV}$ (squares), $0.4 \mathrm{eV}$ (circles), $0.6 \mathrm{eV}$ (triangles) and $0.8 \mathrm{eV}$ (stars). The lines are just a guide to the eyes.

Fig. 3 - Proportion of injected electron-hole pairs that undergo recombination in the PPV layers, with random, parallel and perpendicular morphologies, as a function of the average conjugated segment length, when the zero-field barrier height at both polymer/electrode interfaces is $0.2 \mathrm{eV}$ (squares), $0.4 \mathrm{eV}$ (circles), $0.6 \mathrm{eV}$ (triangles) and $0.8 \mathrm{eV}$ (stars). The lines are just a guide to the eyes.

Fig. 4 - Total number of charges (electrons and holes) that are stored within the PPV layers, with random, parallel and perpendicular morphologies, as a function of the average conjugated segment length, when the zero-field barrier height at both polymer/electrode interfaces is $0.2 \mathrm{eV}$ (squares), $0.4 \mathrm{eV}$ (circles), $0.6 \mathrm{eV}$ (triangles) and $0.8 \mathrm{eV}$ (stars). The lines are just a guide to the eyes. 
Fig.1

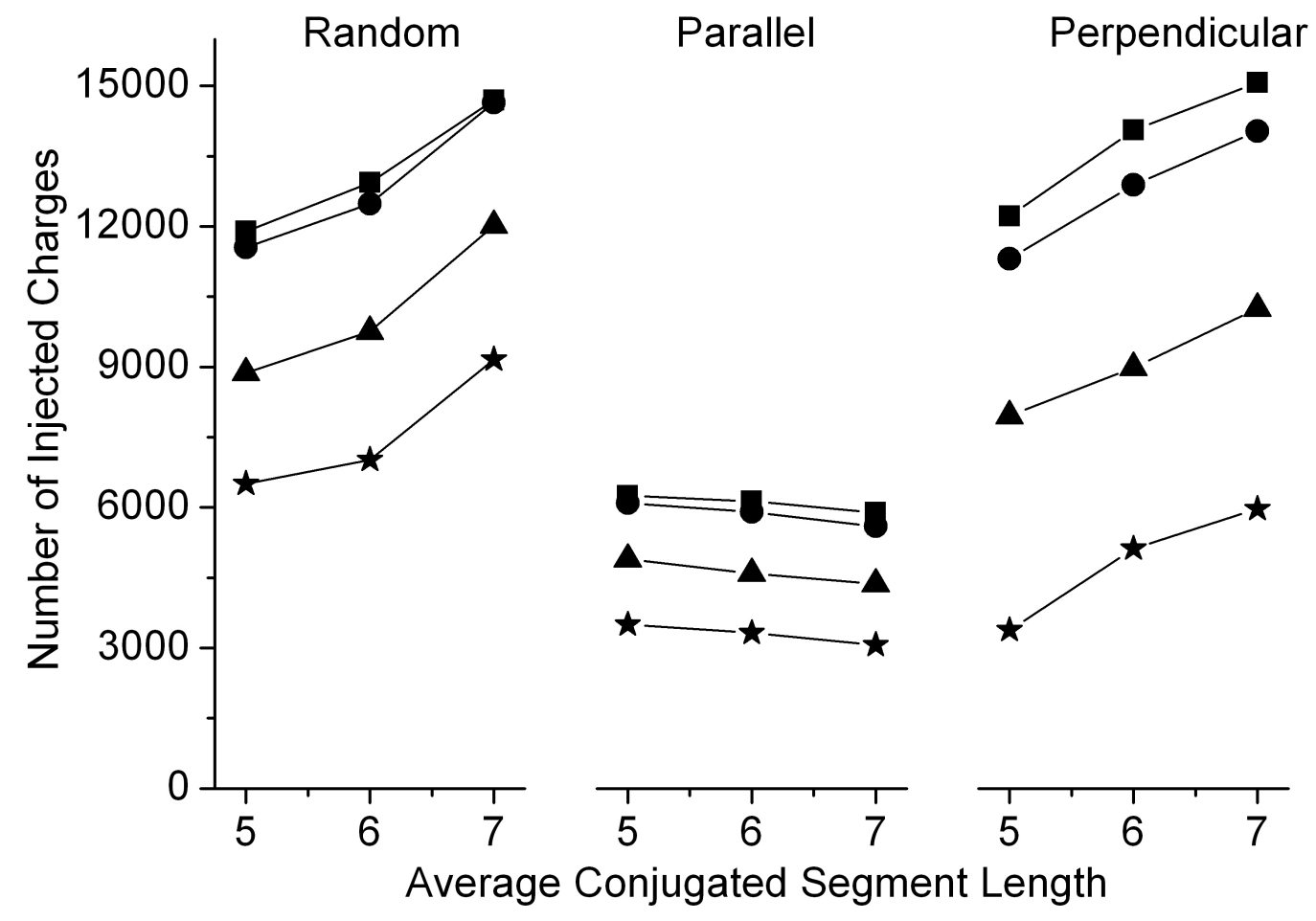


Fig. 2

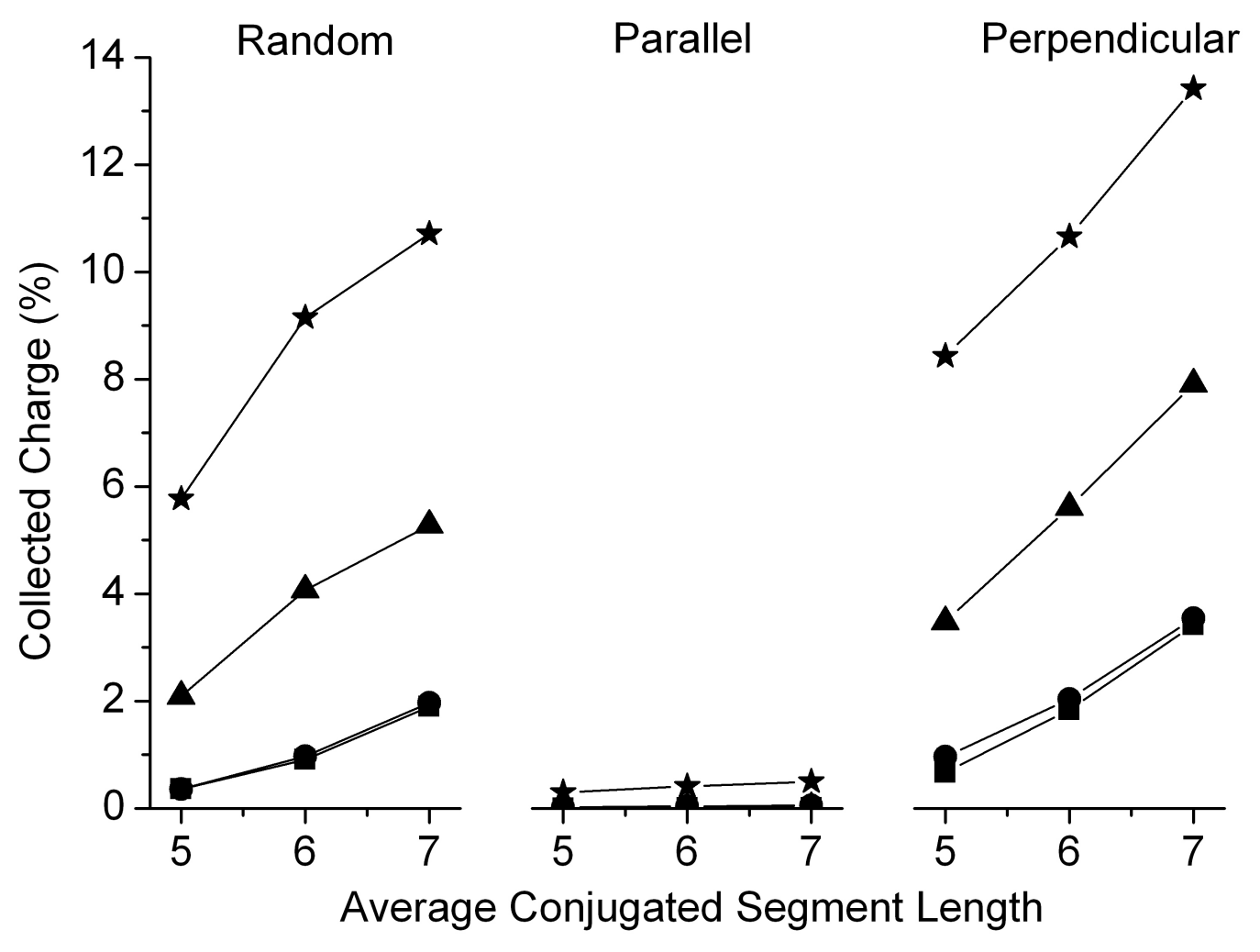


Fig. 3

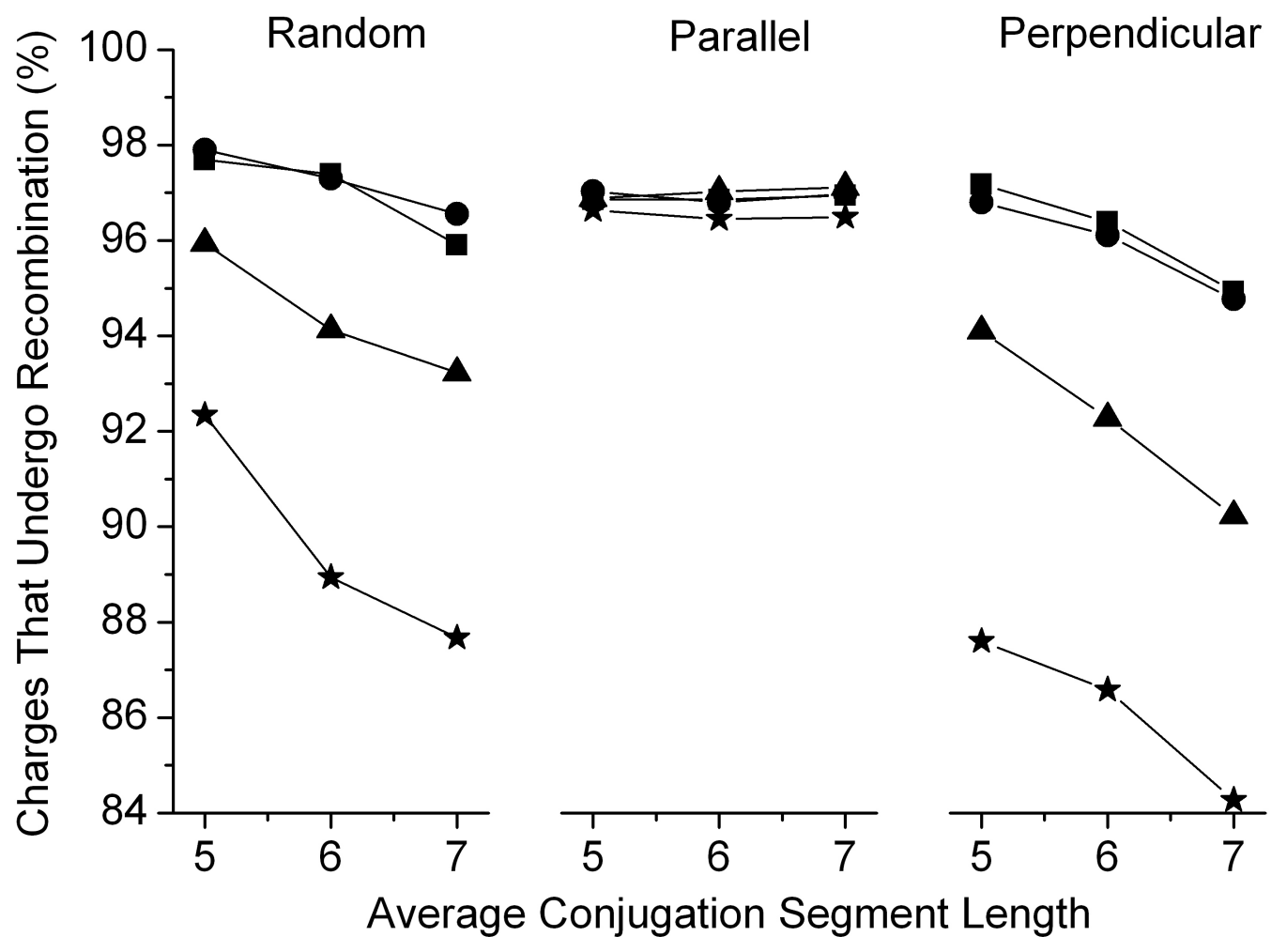


Fig. 4

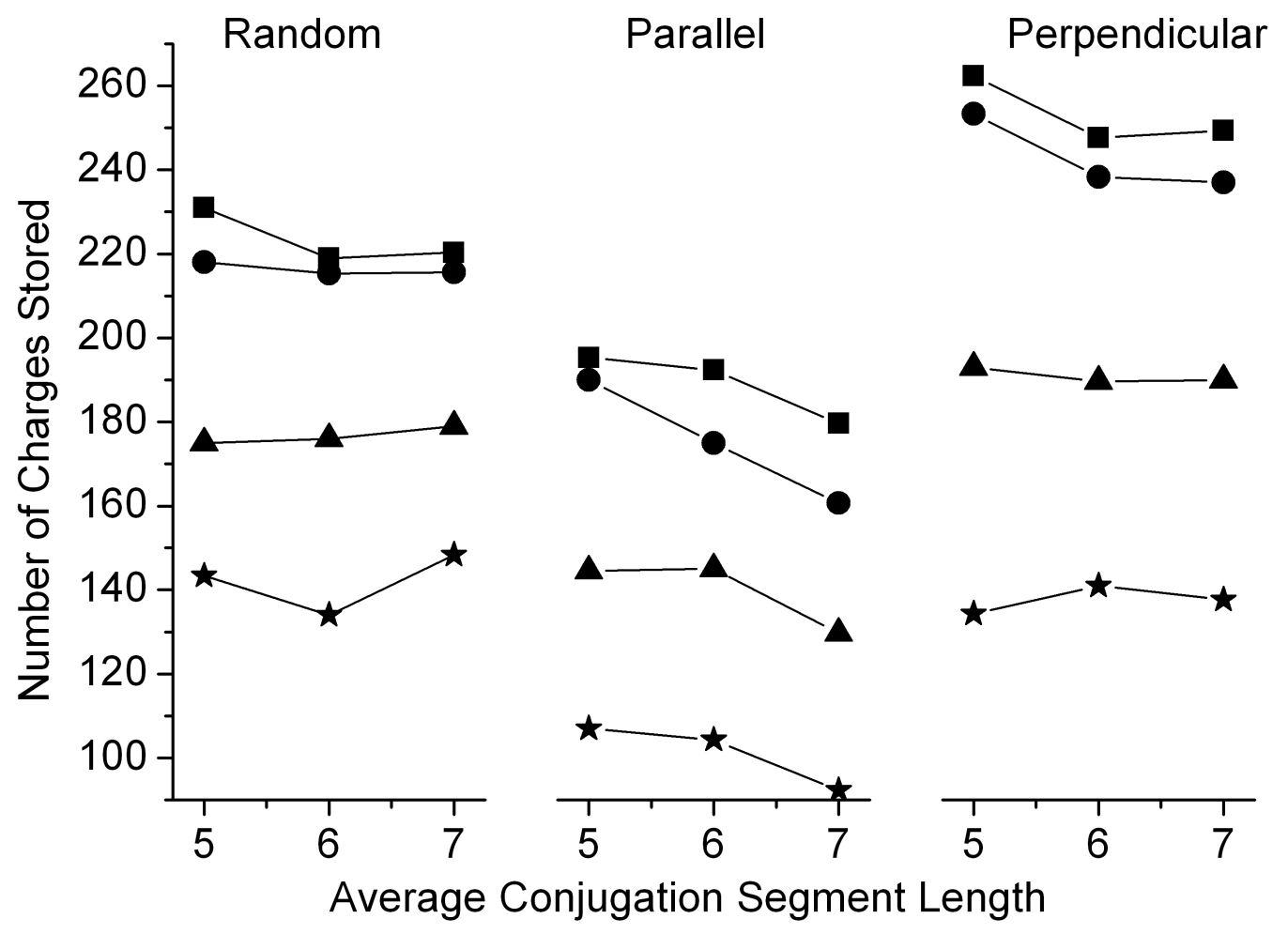

